\title{
PocketAID: The Pocket Atlas of Infectious Diseases Mobile Application
}

\author{
Bonnie Gale, Hamid Mansoor, Chen-Yeou Yu, Lauren E. Charles
}

Pacific Northwest National Laboratory, Richland, Washington, United States

Objective

The Pocket Atlas of Infectious Diseases (PocketAID) mobile application developed at Pacific Northwest National Laboratory (PNNL) provides infectious disease education and decision support offline for an enhanced personal situational risk assessment anywhere in the world. The app integrates a user's location, demographic information, and infectious disease data to present the user with important information including personalized, calculated risk level.

PocketAID features a global disease distribution map and epidemiological curve of country-based case counts by year. Filter options allow users to customize disease lists available to aid in situational awareness. PocketAID, first of its kind, is being developed for offline decision support use by Department of Defense's Defense Threat Reduction Agency (DTRA).

\section{Introduction}

There are a wide variety of available web-based apps, such as CDC's Epidemic Information Exchange, that provide infectious disease information and disease distribution [1]. Publicly available, online data can be used to inform a user of general risks based on disease distribution maps and case count data. Unfortunately, each app contains different aspects of the data, which is often represented in different ways and incompatible formats. This heterogeneity can overwhelm a user with confusing information making it difficult to interpret or gain valuable insight into their own situational risk in a specified location. In addition, online resources do not filter information based on the user's current location or situational needs and, therefore, reduces the value of information a user may be interpreting.

However, information formatted and represented appropriately in a single app could be used to better understand an individual's situational infectious disease risk. In addition, this information may further educate a user based on a situation or incident to prevent disease spread, especially in higher risk populations. To accomplish these goals, PNNL has developed an offline, Android app that provides the user with simple, easy to understand filterable global infectious disease information integrated with their location to provide personalized situational health risk and decision support in the field.

\section{Methods}

This prototype mobile app was a product of PNNL's Biosurveillance Application Competition, sponsored by DTRA. Our implementation of this prototype consisted of two parallel efforts: data collection and Android app development.

Data. Infectious disease information was collected from CDC, WHO, Biosurveillance Resource Directory, and Analytics for Investigation of Disease Outbreaks websites [1-4]. Visualization feature data for global disease distribution and the case count curves was collected from CDC, WHO, and ECDC websites [1,2,5]. Data used for the disease filter and risk level warning features were associated to the collected infectious disease information and user inputted demographic information.

Application. The prototype app was built using Android operating system. Information about diseases, e.g., transmission mode, symptoms, properties, was stored in SQLite database that was imported into the phone at install time to provide offline information access. We used OSMDroid, an open source project, for map and location services. Downloaded map tiles made zoomable, interactive maps available offline.

\section{Results}

PocketAID biosurveillance Android app was targeted for active duty service members, although deemed useful to a much broader audience. Given the various challenges that service members can face during deployment, such as no connectivity in remote are as, 
the app provides full functionality offline. The general purpose of PocketAID is to provide a user with infectious disease situational awareness and decision support, not be used as an analytic tool to test, treat, or diagnose disease.

Upon launch, the user is shown their location on a zoomable, interactive map and a list of diseases that are known to be present in their current country (detected automatically using the device's GPS). The user can change their location by selecting a coun try from the location dropdown menu, filtering the populated list of diseases. The user can further filter diseases by disease attributes: symptoms, transmission, and properties. Clicking on a disease redirects the user to a page with more details about the disease, an interactive map of global disease distribution, and epidemiological curve displaying case counts by year for selected disease in selected country.

The user can input basic demographic information (i.e., age, gender, occupation, and pregnancy status) in the settings page of the app, which then enables an automated assessment of disease risk. Since specific diseases pose an increased risk to certain groups of people, the app can personalize the user's risk level. In other words, if a user's demographic information matches a disea se's risk groups, the user is shown a warning alert.

The app was awarded second prize in the competition by judges from across the government for its perceived benefit to biosurveillance, innovation and originality, quality of user experience, and long-term value and sustainability.

\section{Conclusions}

The PocketAID provides global disease distribution on a zoomable map, infectious disease background information, disease case counts, offline capabilities, and diseases filtered by the location. This educational app offers a situational health risk as sessment for the user through accessing infectious disease information with a disease attribute filter, personalized risk level warning, and user's GPS or selected location to help improve decision support and reduce situational risk. The app was vetted by domain experts across the US Government, who found it to be useful and valuable.

\section{Acknowledgement}

This work was funded by the Defense Threat Reduction Agency (project number CB10190).

\section{References}

1. Centers for Disease Control and Prevention [Internet]. Atlanta (GA): U.S. Department of Health \& Human Services; [cited 2018 Aug 17]. Available from: https://www.cdc.gov/.

2. World Health Organization [Internet]. Geneva (Switzerland): World Health Organization; c2018 [cited 2018 Aug 17]. Available from: http://www.who.int/gho/en/.

3. Margevicius KJ, Generous N, Taylor-McCabe KJ, et al. 2014. Advancing a Framework to Enable Characterization and Evaluation of Data Streams Useful for Biosurveillance. PLoS One. 9(1), e83730. doi:https://doi.org/10.1371/journal.pone.0083730. PubMed

4. Analytics for Investigation of Disease Outbreaks [Internet]. Los Alamos (NM): Los Alamos National Security, LLC for the U.S Dept. of Energy's NNSA; c2018 [cited 2018 Aug 17]. Available from: https://aido.bsvgateway.org/.

5. Surveillance Atlas of Infectious Diseases [Internet]. Solna (Sweden): European Centre for Disease Prevention \& Control; c2018 [cited 2018 Aug 17]. Available from: http://atlas.ecdc.europa.eu/public/index.aspx. 

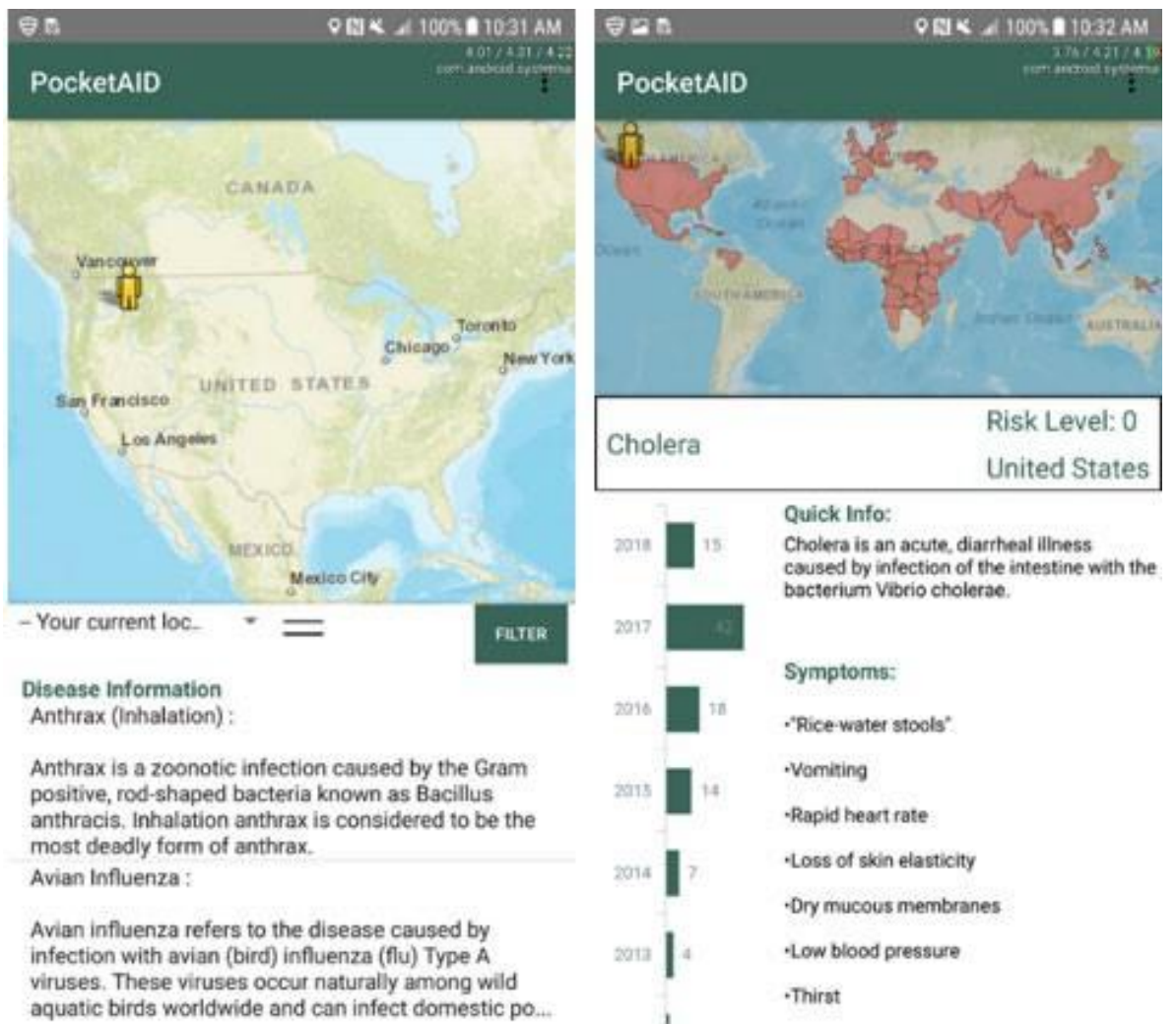

Figure 1. Application prototype main page (left) and disease information page (right). The main page includes filtered diseases by the user's current location or a selected location from a drop-down menu and further filtering by disease transmission, properties, or symptoms. Disease information pages include a risk level warning dialogue box, global disease distribution on a map, epidemiological curves, and disease information. 